\title{
IdeAs
}

Idées d'Amériques

\section{Les dilemmes des universités interculturelles au Mexique}

Dilemas de las universidades interculturales de Mexico

Dilemmas of intercultural universities in Mexico

David Lehmann

\section{OpenEdition}

\section{Journals}

Édition électronique

URL : https://journals.openedition.org/ideas/331

DOI : 10.4000/ideas.331

ISSN : 1950-5701

Éditeur

Institut des Amériques

Référence électronique

David Lehmann, "Les dilemmes des universités interculturelles au Mexique », IdeAs [En ligne], 2 |

2012, mis en ligne le 22 juin 2012, consulté le 19 octobre 2022. URL : http://journals.openedition.org/ ideas/331 ; DOI : https://doi.org/10.4000/ideas.331

Ce document a été généré automatiquement le 19 octobre 2022

\section{(i) $9 \Theta$}

Creative Commons - Attribution - Pas d'Utilisation Commerciale - Pas de Modification 4.0 International - CC BY-NC-ND 4.0

https://creativecommons.org/licenses/by-nc-nd/4.0/ 


\title{
Les dilemmes des universités interculturelles au Mexique
}

\author{
Dilemas de las universidades interculturales de Mexico \\ Dilemmas of intercultural universities in Mexico
}

\section{David Lehmann}

David Lehmann agradezco a la British Academy por su apoyo a la investigación que subyace este trabajo, así como a Capucine Boidin por sus valiosos comentarios.

1 A la diferencia de lo que puede suceder con temas como la democracia o el concepto de etnicidad, la interculturalidad y su pariente el multiculturalismo, más que conceptos, son términos descriptivos. En ellas se cruzan las categorías de cultura, religión, patrimonio y lenguaje, y las esferas normativas de reconocimiento, justicia e igualdad. La mera idea de distinguir entre culturas o de trazar líneas divisorias entre ellas, depende de factores locales y subjetivos como el uso de idiomas y su variable papel como marca identitaria, la adherencia a ciertas prácticas religiosas, el uso de cierto ropaje o el fenotipo.

2 Así que prefiero partir de conceptos como ciudadanía, igualdad y justicia social, para después analizar cómo distintas variables culturales son movilizadas en su defensa o en su detrimento. Por ejemplo, mientras la democracia o la justicia social pueden ser fortalecidas por medidas como la prohibición del velo entre jóvenes estudiantes de colegio, en otros casos tal efecto no sería de esperarse; o mientras en algunas situaciones la educación bilingüe favorece la igualdad de oportunidades, en otros es irrelevante.

3 Este enfoque, además, nos ayuda a evitar caer sin quererlo en juicios de valor sobre una $\mathrm{u}$ otra costumbre o rasgo cultural perteneciente a uno $\mathrm{u}$ otro grupo. Porque al tomar posiciones sobre el reconocimiento debido del otro, nos encontramos a menudo en la disyuntiva de decidir si algún rasgo o práctica merece respeto en sí mismo. Claro que no admitimos que esto sea un criterio, pero el riesgo de que caigamos en tales juicios de valor no es de ninguna manera despreciable; hasta es inevitable. Piense en las controversias en torno a los derechos de las mujeres en ciertos contextos donde se han 
abierto espacios - electorales o educacionales - a instituciones fundadas sobre bases otras que las republicanas o las liberal-democráticas: en un municipio del estado mexicano de Oaxaca, por ejemplo, amparándose en la legislación que introdujo los usos y costumbres en las elecciones locales, las mujeres fueron excluidas de la participación política, aunque probablemente el caso haya sido resuelto posteriormente sin tanta publicidad como la denuncia (Sierra 2009).

Otro aspecto que caracteriza las discusiones antropológicas y sociológicas sobre estos temas tiene que ver con la multiplicidad y la superimposición de roles que, por lo menos en las concepciones más convencionales de la investigación, suelen estar separados. Los debates en torno a la interculturalidad se dan en un foro heterogéneo que reúne activistas, gestores, ONGs, políticos, universitarios, representantes y asesores de asociaciones, movimientos indígenas e instituciones internacionales con sus funcionarios y asesores.

5 Esto en sí no sería nada diferente de muchos debates, si no fuera por el hecho de que los individuos acumulan roles en distintas esferas e instituciones, dirigiéndose a públicos variados y mezclados. Están en juego relaciones de influencia, quizás de poder, entre intelectuales/consultores y líderes o militantes de los movimientos que dependen de recursos externos, no sólo por razones materiales sino también para alcanzar y mantener cierto nivel de credibilidad, frente a interlocutores nacionales $e$ internacionales.

6 Un ejemplo del laberinto que comportan estas dificultades es el notable libro de Joanne Rappaport titulado Utopías Interculturales (Rappaport 2005; Rappaport 2008) cuya autora navega sin dificultad entre sus múltiples posicionamientos y roles ${ }^{1}$. De alguna manera, el papel de analista o intérprete, es compartido con su red de colegas-amigosinformantes. Sin embargo hay una asimetría de poder que no se debe olvidar: los que escriben y publican en inglés tienen un auditorio internacional mucho más amplio y es probable que tengan la posibilidad de influenciar decisiones tomadas en agencias de desarrollo en un mayor grado que sus interlocutores locales.

El presente trabajo también es producto de cierta superimposición de roles, aunque no de una inmersión tan prolongada como la de Rappaport. Las entrevistas concedidas han sido en gran parte con colegas en las Universidades Interculturales y en instancias de gestión gubernamental que yo visité no simplemente como investigador sino también como colega, o durante un Congreso internacional sobre Educación Superior para Pueblos Indígenas (Pátzcuaro, México, noviembre 2009) donde también presenté un trabajo científico. La relación entablada en esos contextos con los entrevistados no es lo mismo que con un informante escogido al azar o aun con una persona conocida en el curso de una etnografía en profundidad, porque el investigador tiene que tomar en cuenta la posibilidad de que ellos lean o se interesen en lo que publique. Pero la desventaja que eso pueda acarrear, en términos de cierta cautela al publicar, se compensa por la ventaja de la confianza mútua profesional, aún si esta confianza no es siempre absoluta, como bien sabemos. En el campo de la interculturalidad, las discusiones pueden influenciar por ejemplo las políticas de las ONGs que muchas veces financian a las instituciones interculturales, o pueden estar ligadas con decisiones sobre el contenido de la enseñanza en escuelas o universidades interculturales, aunque no es mi propio caso. Pero no se trata sólo de intereses creados: estas discusiones involucran elementos subconscientes y la psicología de los participantes, que son por un lado imposibles de ignorar pero por otro lado imposibles de traer a luz porque eso 
amenazaría el tono impersonal que es consubstancial a la coexistencia académica. Además son temas que tocan las mismas relaciones entre los participantes, porque cada uno sabe que su contribución es producto, en algún grado, de su propia identidad nacional o étnica o de las construcciones e interpretaciones que él o ella misma da de esa identidad, o de los esfuerzos que hace para separarla de su actividad profesional; y eso sin contar las construcciones que los otros realizan de nuestra identidad o de los esfuerzos que hacemos para esconderla, controlarla o trascenderla, ni el trabajo de los otros para construir o reconstruir su propia identidad. Y careciendo de una definición adecuada de la palabra 'identidad' nos refugiamos en infinitas discusiones sobre las identidades construidas o inventadas, no porque la identidad no exista sino porque, por aversión a cualquier veleidad 'esencialista' hay una renuncia perfectamente comprensible a admitir que una persona pueda estar condenada a vivir una identidad única y homogénea. Lo curioso es, sin embargo, que la misma antropología que huye del esencialismo también aboga a veces por la recuperación de tradiciones y cosmovisiones indígenas y reclama la apertura de espacios institucionales para ellas, notablemente en la educación, haciendo caso omiso de los cuestionamientos antiesencialistas o constructivistas. Sería fácil ironizar sobre estos posicionamientos polifacéticos, como lo hacen los críticos del multiculturalismo, intelectuales liberales muchas veces egresados de la universidad del marxismo (Roger Bartra en México por ejemplo; Slavoj Žižek en Europa) (Bartra 1999; Žižek 1997)² pero la ironía simplemente permite esquivar el problema real de la inclusión y sus dimensiones culturales y linguísticas.

8 A la luz de este trasfondo, este artículo presenta entrevistas y textos que tomados en su conjunto, muestran las diversas interpretaciones que profesionales hacen de lo que es la educación intercultural y bilingüe (EIB) así como la diversidad de los intereses políticos y burocráticos, que convergen sobre el proyecto del Estado mexicano de construir un sector de Universidades Interculturales (UI).

Voy a referirme a cuatro tipos de información: primera, la documentación oficial sobre educación intercultural y bilingüe; segunda, la defensa de puntos de vista expresados en entrevistas y en publicaciones por dos personas reconocidas que representan posiciones de apoyo matizados de dudas y hasta angustias frente a lo que podría llamarse posiciones duras derivadas del posmodernismo o de concepciones que enfatizan la intraducibilidad o inconmensurabilidad de una cultura para otra, sobre todo de culturas indígenas para culturas modernas y hegemónicas. De allí paso a destacar algunos aspectos de entrevistas con profesores en Universidades Interculturales recogidas en varias ocasiones entre diciembre de 2006 y mayo de 2008. Con esto quiero primero mostrar la diversidad de la EIB, y como ella se estructura en formas variadas según el contexto y según la estructura institucional y sus fuentes de apoyo. La EIB es una idea general que se moldea y se adapta según las circunstancias.

\section{Dos versiones oficiales}

10 El gobierno de Vicente Fox creó la Comisión de Coordinación de la Educación Intercultural y Bilingüe (CGEIB) en 2001 y nombró como su jefe fundador a la conocida educadora Sylvia Schmelkes. En 2004 la CGEIB publicó el documento titulado Políticas y fundamentos de la educación intercultural bilingüe en México $(2004)^{3}$, redactado principalmente, según uno de sus autores, con vistas al sector de la educación primaria, 
aunque el texto hable de la educación en general. Lo que llama la atención en este documento es su inspiración en ideas posmodernas y una versión bastante radical de lo que conllevan las diferencias culturales. En este texto de 27 páginas la palabra diversidad viene utilizada por lo menos 45 veces (sin contar trechos citados de otras fuentes ni su uso en significados otros que la diversidad cultural); la palabra otro en el sentido de 'mi otro' o 'el otro de uno' 15 veces y otredad 3 veces. Dos otras palabras de frecuente uso en la literatura posmoderna que aparecen con frecuencia son lógica (16 veces) y epistemología (13). En cambio, las palabras inequidad, y justicia o injusticia son usadas cada una 9 veces.

11 Después de exponer fundamentos formales y legales, el documento se explica sobre la interculturalidad en un estilo más académico que burocrático. Los temas principales son la afirmación de la identidad y el reconocimiento de la diferencia como formas de lucha contra la injusticia. Además el documento va más allá e insiste sobre 'otras lógicas igualmente válidas' y la necesidad de oponer conceptos científicos fundamentales con los derivados de otras culturas 'para encontrar la lógica de los diversos temas de la ciencia.' Y sigue: 'Es necesario analizar y sacar a la luz los etnocentrismos para liberar a cada una de las ciencias de una mirada deformante e innecesaria.' Se invocan las contribuciones de las culturas indígenas que son fundamentales para la ciencia moderna como la 'herbolaria, la astronomía, los tipos de suelo, los ciclos lunares y sus relaciones con el trabajo humano', y se argumenta que 'quizá la contribución más relevante haya sido el ampliar el horizonte de las posibilidades lógicas y de rutas alternas para conocer el mundo en que se habita.' El diálogo intercultural conlleva llegar a nuevas síntesis cognitivas que, a su vez se cuestionan por la historicidad de otras culturas. En nuestro caso (el de la EIB) esta dimensión epistemológica intenta articular las lógicas de construcción de las cosmovisiones indígenas de México y envuelve una mirada amplia y compleja sobre el proceso de construcción del conocimiento, en que los elementos naturales y simbólicos se imbrican...'

12 Al leer este documento parecería que el estado ha tratado de plasmar una concepción de la educación fundada en una idea elaborada de las culturas indígenas y de la diferencia cultural, y una propuesta fuerte de recuperación, de reconocimiento y de valorización de ellas. Pero el documento mucho más corto y programático sobre las Universidades Interculturales ('Proyecto Universidad Intercultural') ${ }^{4}$ adopta un tono muy diferente. Insiste mucho menos sobre la política del reconocimiento y no hace mención de las distintas epistemologías o lógicas. El énfasis es sobre el desarrollo y el acceso y si bien reconoce la incorporación de 'nuevas perspectivas de desarrollo profesional y científico que conjugarán saberes y conocimientos desde diferentes perspectivas culturales' no da tanta importancia a este elemento como en el documento citado anteriormente. Más bien busca 'una interacción creativa y enriquecedora entre diferentes culturas' y proyecta 'una nueva forma de integración de los jóvenes indígenas a los estudios superiores'.

13 Así que en los documentos escritos hay propuestas y sensibilidades diversas, las cuales probablemente corresponden menos a diferencias entre los responsables que a las exigencias de la coyuntura política. El proyecto de Educación superior intercultural es patrocinado por los gobernadores de estados y por la Comisión de Desarrollo de los Pueblos Indígenas. La CGEIB en sí es un organismo coordinador 'horizontal' que no tiene fondos propios para implementar sus ideas. Y de hecho, los programas concretos 
en las universidades tienen un esquema común de carácter aplicado que obedece a expectativas en el mercado de trabajo y a las exigencias del famoso PIFI - Programa Integral de Fortalecimiento Institucional. El PIFI inspecciona y certifica a todas las universidades públicas y sin su certificación, las instituciones pueden perder el derecho de llamarse universidades. Por eso, talvez el currículo estándar de las UI, tiene un perfil orientado más al mercado de trabajo que a temas culturales. Aún así, como veremos, eso no implica necesariamente que en ellas no se estimule una concientización cultural. Los cursos y las carreras suelen concentrase en los campos de Lengua y Cultura Indígena, Turismo Alternativo y Desarrollo Regional Sustentable, con algunas variantes.

Aparte del contenido de las carreras, las UI tienen una importante dimensión de acción afirmativa. Como dice el 'Proyecto' el mero hecho de su ubicación en zonas de población indígena (citando la demanda de pueblos otomíes y mazahua 'por una universidad cercana geográfica y culturalmente' a sus pueblos) facilita el acceso, pero no es necesariamente una garantía de contenido intercultural.

\section{Dudas e incertezas en torno a la educación intercultural}

15 Hay que separar las dos dimensiones - interculturalidad y acción afirmativa. La propuesta del 'Modelo' es de ésta última: modificar la oferta y las condiciones de la oferta de educación superior para aumentar las posibilidades de acceso entre sectores definidos - en este caso, poblaciones indígenas. En principio - pero sólo teóricamente la acción afirmativa no necesita, ni siquiera, mencionar a una preferencia racial o étnica porque no selecciona directamente según esos factores: son los mecanismos indirectos diseñados para que estudiantes indígenas aspiren y tengan acceso a la educación superior.

Sin embargo, en el campo de la educación, la interculturalidad propone objetivos menos precisos tocando temas más sensibles y controvertidos que a veces afectan hasta la intimidad de la persona: la capacidad de expresarse en un idioma sin ser menospreciado por el acento o el idioma mismo; la ansiedad de pensar que la ropa que uno lleva, o su fenotipo, sean motivo de desprecio por otros; el miedo (mucho menos presente en América Latina que en otros lugares) a que ritos considerados consubstanciales al ciclo de la vida puedan ser vistos como inhumanos o absurdos por poderes estatales y poderosas corrientes en la opinión pública.

Pero en el campo del lenguaje, la interculturalidad cruza lo íntimo y lo cotidiano con el tema de la exclusión. Se trata en gran parte, aunque no exclusivamente, de una cuestión técnica: ¿cómo ayudar a niños y jóvenes que han sido educados en su hogar en una lengua indígena o no hegemónica a desarrollar capacidades lingüísticas compatibles con el estatus de profesionales competentes? Es el tema pretérito de linguistas como Luis Enrique Lopez (Dutcher 2002; Lopez 2005; Lopez 2009). Aparte de esta cuestión técnica existe una idea más implícita que desarrollada, según la cual la lengua materna es parte consubstancial de la identidad de una persona y que negarle el reconocimiento de su idioma como medio de comunicación digno, es vulnerarle en su persona. Esta concepción es aplicable también en situaciones donde la lengua materna comparte el espacio comunicativo con la hegemónica, aún en el contexto íntimo de los hogares o de las comunidades herederas de un patrimonio cultural-lingüístico propio. 
18 Ahora bien, restaurar el uso cotidiano de un idioma es tarea compleja porque involucra no sólo la educación, sino las costumbres subconscientes de la vida social. Sin embargo, cuando se trata de poblaciones históricamente marginadas, y cuya marginación, por mucho que se base en dinámicas socio-económicas seculares, tiene también un fuerte componente cultural, reanudar los hilos que los liguen a su pasado, creando las condiciones para que sus hijos tengan conciencia y orgullo de ella, no puede ser otra cosa que positivo: el problema para los educadores es qué peso dar a este aspecto de los programas educativos. Enseñar en escuelas rurales un idioma que no es escrito a niños cuyos padres, por mucho que sean estén orgullosos de su patrimonio lingüístico, son a veces escépticos en cuanto a la utilidad de su retransmisión como conocimiento erudito u oficial, es difícil y costoso - aún en territorio zapatista (Baronnet 2011). Requiere fuertes inversiones en formación de profesores, en materiales etc. Sobre todo necesita un compromiso mantenido por muchos años. Si a eso se añade el desafío de la renovación lingüística en la vida cotidiana, la tarea se hace aún más compleja. Esto está comprobado en el caso de Bolivia, donde los sindicatos de profesores mostraron una fuerte resistencia a la EIB (Noel 2006; Lopez 2009) y del Perú donde, a pesar de la dinámica liderada por FORMABIAP en la región de la Selva, los expertos concuerdan con el panorama desalentador de la Sierra (Lopez, 2009 citando a Lucy Trapnell).

19 Si bien los lingüistas argumentan a favor de la educación bilingüe, los antropólogos tienden a mostrar escepticismo, fundado en sus trabajos de terreno, o desplazan su entusiasmo al campo de la cultura en un sentido más general, de las cosmovisiones, como en el caso del CRIC en Colombia (Rappaport 2008). Este entusiasmo acaba plasmándose como un apoyo a los movimientos étnicos de inspiración cultural como una especie de lucha ventrílocua por la ciudadanía en la cual la substancia cultural es un vehículo de una lucha por derechos - derechos a la tierra, a la autonomía política - y por la inclusión social en forma de educación, empleo, e igualdad. Así por ejemplo un prominente defensor de la causa indígena, Guillermo de la Peña, da poca importancia a la educación bilingüe (de la Peña 2005).

Miremos ahora los pensamientos de dos figuras dedicadas a la educación intercultural. Antonio Saldívar es un educador con doctorado de la Universidad de Salamanca y fuertemente influenciado por el psicólogo constructivista ruso Vygotsky, cuya obra, junto con la de Paulo Freire y de Jerome Bruner, es uno de los pilares de la educación liberadora. Después de 14 años trabajando en la investigación sobre temas de desarrollo en el Colegio de la Frontera Sur, fue nombrado Director del INED (Innovación Educativa) - una institución apoyada conjuntamente por El Colegio y el gobierno de Chiapas (Saldívar 2001). En una entrevista realizada en mayo de 2008 expresó las tensiones culturales y hasta psicológicas que radican en la extrema desigualdad y la imposibilidad de resolverlas.

21 Con franqueza, Saldívar contó cómo una persona como él, al llegar a una comunidad indígena, no puede evitar una sensación de jerarquia: 'de entrada hay una sobrevaloración de lo que yo puedo aportar a esa comunidad y hay una subvaloración de esta comunidad'. El habla de su llegada no como individuo sino como portador de un proyecto de mejoramiento - siempre el profesional llega con un proyecto. El que viene de fuera, si es un profesional, entabla de entrada una relación de desigualdad, de poder, que niega la posibilidad de diálogo en el sentido de conversación entre iguales: 'yo no puedo dialogar con el otro...porque entonces no se va a dar un proceso realmente de aprendizaje entre ambos'. Eso viene también de sus hábitos, de su modo de hablar, de 
vestirse y de su red social. Iniciativas como las UI le parecen contribuir a acentuar la desigualdad específicamente cultural, o por lo menos no reducirla. Para él, además, el currículo de las UI es un buen ejemplo de la misma asimetría, por su orientación al mercado, que tiende a folclorizar las culturas.

Al mismo tiempo, sin embargo, Saldívar era claramente consciente de que las familias indígenas esperan que los estudios confieran a sus hijos las capacidades - notablemente lingüísticas - que requieren para entrar al mercado laboral. 'Claro ... piensan que el tzotzil ya no les sirve...'; ellos reconocen las desigualdades enraizadas en su entorno, pero Saldívar ni siquiera está convencido de que las políticas y los proyectos puedan revertir la tendencia, porque están tan imbuidas ellas mismas de la relación de desigualdad como la que él describe para su propia persona.

Saldívar habló también de la distancia cultural, pero en términos distintos de las usadas por el documento-matriz de la CGEIB. Esbozó el contraste entre el espíritu occidental de 'pensamiento abstracto, de sistematización y clasificación' y el énfasis indígena en el aprendizaje por el contacto personal. No hay una sola manera de adquirir el conocimiento, pero al mismo tiempo para los que poseen una cultura mestiza o dominante no hay interés en adherir a una identidad cultural con nombre: eso es sólo para 'el otro... el otro oprimido': : '...yo nunca me cuestiono quién soy yo... el que tiene que cuestionarse y tratar de entender su cultura es el otro, yo no. Entonces cuando de repente me veo frente a esta circunstancia hay una incomprensión'. De hecho, talvez la misma atribución de la necesidad de proferir una identidad cultural es una especie de imposición externa de 'mi' o 'nuestra' parte.

24 En esta frase laberintina, el 'yo' de Saldívar es la tercera persona - un representante estereotipado de la cultura dominante. No es él mismo porque si fuera él no estaría hablando de esa manera porque no reconocería estas contradicciones. Pero al mismo tiempo está diciendo que, lo quiera o no, sí, él es esa otra persona - esa persona que coloca el 'otro' - el indígena - en una situación donde, de nuevo, aunque no lo quiera, es heredero de una cultura y tiene de alguna manera que responder por ella y dominarla. La cultura dominante está exenta de auto-comprensión o auto-descripción: su capacidad de autocrítica (poscolonial, universalista etc.) es otra afirmación más de su status como cultura hegemónica en manos de una elite.

Con todas sus dudas, Saldívar también se dedica a tareas prácticas. En 2004 publicó en la Revista Mexicana de Investigación Educativa (Saldívar Moreno, Micalco Mendez et al. 2004) una descripción de un curso titulado 'Diplomado en Educar en y para la diversidad' organizado para profesores. Eran casi todas mujeres, ya que las llama de 'maestras' - de primaria durante el ciclo escolar 2001-2002. Se inspiró en el método constructivista de las obras de los psicólogos Vygotsky y Jerome Bruner: 'el trabajo tiene que partir de la experiencia personal, dado que el aprendizaje opera a nivel del cuerpo y no sólo de la cabeza como estamos tradicionalmente acostumbrados a pensar'.Aunque no hace mención directa de Paulo Freire, el espíritu de éste impregna todo el texto. Por ejemplo, al hablar de la construcción de 'nuevos aprendizajes con base en los conocimientos previos, desarrollar nuevos conocimientos utilizando como marco referencial la realidad de los sujetos' el artículo demuestra claramente la influencia Freiriana. ${ }^{5}$

El propósito del enfoque constructivista, según el trabajo de Saldívar y sus co-autores, es la apropiación de 'las estrategias pedagógicas participativas' y de hecho el trabajo muestra que así fue, según una evaluación provisional. Pero el efecto no fue muy 
duradero : de vuelta a la rutina de sus clases, las maestras se vieron presionadas para volver a sus hábitos anteriores. La experiencia viene incrementada por una investigación llevada a cabo en 2007 con estudiantes de la flamante Escuela Normal Indígena Intercultural Bilingüe «Jacinto Canek» (ENIIB) en las afueras de San Cristobal de las Casas (Navarro Martinez and saldívar Moreno 2011-2012). En ésta última se vio que si bien, los estudiantes absorbieron el credo intercultural, en el sentido de apreciar la importancia del diálogo de culturas, con la apreciación de la diversidad de lenguas indígenas habladas entre ellos y la pérdida de la vergüenza de hablar en idioma indígena en público, la interculturalidad queda como una burbuja dentro de la institución educacional. Este estudio nos alerta que educar en forma bilingüe no garantiza la dimensión intercultural y entonces puede dejar intocadas las relaciones de poder y de menosprecio. Por otro lado también ve como insuficiente el reconocimiento ofrecido por la multiculturalidad. En la óptica intercultural, el reconocimiento mutuo debe ir de par con el reconocimiento de las condiciones en que se establecen las formas de interacción entre herederos de distintas culturas: 'no es suficiente sólo el reconocimiento. La interculturalidad cuestiona cómo en una situación de relaciones marcadas por una profunda asimetría se puede establecer un diálogo constructivo, un diálogo que nos enriquezca mutuamente'. Hemos aquí el punto débil de los que tanto insisten e invierten en lo bilingüe. Concluye recordando que la interculturalidad conlleva reconocimiento mútuo:

Es necesario ligar la educación al contexto inmediato, problematizando la realidad y proponiendo acciones inmediatas dirigidas a la sociedad mestiza, pues de lo contrario estaremos hablando de una educación indígena bilingüe, pero no intercultural.

Saldívar expresa las tensiones provocadas por el encuentro entre la teoría intercultural y el desafío de la educación intercultural. Es posible que eso explique la brecha entre los dos documentos de la CGEIB citados arriba - el primero apuntando al reconocimiento de la diferencia y de las diversas epistemologías y cosmovisiones, y el segundo más enfocado sobre los programas concretos en las UI. También vemos las dificultades de la antropología frente a las exigencias encontradas de la interculturalidad, y de las pretensiones de aparatos estatales que buscan al mismo tiempo ensalzar a la interculturalidad y encauzarla de acuerdo a sus propios proyectos de desarrollo o de convivencia social. En este caso también la antropología comprometida se ve obligada a matizar su defensa de la otredad, de lo indígena, y de lo poscolonial, para no caer en 'esencialismos' por un lado y para evitar la defensa de prácticas indeseables sobre todo cuando se trata de los derechos de las mujeres (Walsh 2000; Walsh 2002; Sierra 2009). Además se nota en los textos de estas figuras destacadas en los debates en torno a la interculturalidad, un cuidadoso afán de no dejar que la preocupación con cuestiones culturales oculten o marginan a los temas de poder y de desigualdad. Más bien parecería que están tratando de cernir el lugar de la diferencia cultural y de la recuperación identitaria en el contexto de esas luchas. Pero ese afán las deja en un espacio indefinido, que no importaría, si no fuera que la discusión en torno al universalismo es candente y tiene consecuencias importantes en el diseño de cursos en todos los niveles de la educación. Así citemos a Sierra:

Desde la perspectiva de la interculturalidad, la diversidad es un valor que debe acompañar al reconocimiento de lo propio, al mismo tiempo que hace relevante la diferencia colonial, es decir,

el hecho mismo de la subordinación y el poder en los que se han construido históricamente esas diferencias. La interculturalidad apuesta también a construir 
diálogos, pero desde nuevos contextos que reconozcan las injusticias históricas que han marcado la vida de los pueblos indígenas y otros grupos minorizados (p.76)

Por otra parte la interculturalidad, según Sierra,

ofrece también un enfoque sugerente para debatir las maneras en que discursos producidos en otros contextos, como los referidos a los derechos humanos y los derechos de las mujeres, puedan discutirse desde la diferencia colonial; esto es, haciendo relevante la condición histórica de subordinación de las mujeres indígenas como parte de sus pueblos, pero discutiendo también las visiones universalistas que conlleva el concepto mismo de los derechos humanos de las mujeres, para evitar reproducir visiones homogéneas de lo que significa ser mujer en contextos culturales diversos. (ibid.)

Lo que está en duda es si se puede dejar estas dos concepciones lado a lado. Catherine Walsh exhibe la misma propensión por juntar posiciones que si no son exactamente contradictorias, evocan diferentes enfoques y dejan a los lectores y a los militantes que se inspiran en sus ideas, escoger su propio plato de este menu 'à la carte'. Así Walsh aboga por:

procesos de interculturalización de vía mútiple...[que\} necesariamente implican procesos de desubalternización y decolonialización... dirigidos a fortalecer lo propio como respuesta...frente a la violencia simbólica y estrutural (Walsh 2002)

Lo que le preocupa a Walsh - o le preocupaba en el 2002, ya que ha escrito mucho después ${ }^{6}$ - es que 'la lógica multicultural del capitalismo global parte de la diversidad etnico-cultural y no de la diferencia colonial' - argumento que retoma el 'ex-abrupto' de Zizek (Zizek 1997). Quiere un 'universalismo plural e incluyente' al mismo tiempo que una 'interculturalidad ...contra-hegemónica y de transformación'. La ambiguedad no es de sorprender ya que ella quiere englobar tanto a la población negra o afroecuatoriana como a los indígenas en una interculturalidad contestatoria de un orden colonial, y por eso la palabra 'cultura' tiene que ser usada en una acepción muy amplia.

31 Walsh, como Sierra, quiere escapar del binomio 'particular-universal' o 'relativistauniversalista'. Estos escritos sufren de ambigüedad porque se dirigen a múltiples públicos, entre ellos sobre todo al académico, preocupado por precisión conceptual, y al militante, preocupado por guardar su distancia frente al esencialismo, al neoliberalismo, a las instituciones multilaterales como el Banco Mundial, y a los gobiernos. (Los dos públicos pueden perfectamente coexistir en una sola persona.) A aquéllos les dicen que su universalismo tiene a menudo connotaciones coloniales, y a éstos les dice que hay que evitar los excesos, descritos por Walsh misma, en una frase que hace alusión a roces entre movimientos negros e indígenas como 'actitudes y prácticas racializadas y discriminadoras dentro de los movimientos y organizaciones'.

A la luz de estas dificultades, escuchemos de nuevo la voz de Saldívar, porque reconoce que él mismo, tal como Sierra, Walsh y Rappaport, no puede sino reconocerse como parte en el proceso (en el sentido jurídico). Es evidente que la discriminación tiene múltiples dimensiones culturales: el lenguaje, el acento, el idioma, el lugar donde uno vive, el color de la piel, la ropa, se conjugan con poder, dinero y status profesional en el complejo colonial al cual alude Walsh. Pero estos mismos elementos simbólicos o culturales pueden reproducirse en el movimiento indígena, con sus intelectuales, sus profesionales, sus ONG, y su dependencia de los organismos e instituciones internacionales.

Por otra parte hace falta desmenuzar los elementos culturales de la dominación (o del colonialismo en el lenguaje de la tendencia decolonial) y evitar ese mismo error de 
empaquetar etnicidad y cultura, o lengua y etnicidad, porque en cada situación ellos juegan un papel distinto en la producción y la reproducción de la dominación y la exclusión. Aprender su propio idioma como lengua escrita, por ejemplo, cuando esa lengua no tiene tradición escrita, puede ser visto como la imposición de un modelo hegemónico de lo que es una lengua 'de verdad'. Enseñar el idioma hegemónico como lengua extranjera en la escuela primaria puede a la vez mostrar respeto cultural y tambien mejorar la calidad de la enseñanza, pero para eso hacen falta profesores capaces de hablar el idioma de los alumnos.

Por otra parte el llamado a un 'universalismo plural e incluyente' me parece a mí diferenciarse poco o nada de un llamado al ejercicio de derechos humanos y civiles republicanos y universales. La cuestión de la autodeterminación no es una cuestión cultural, y si se trata de un criterio étnico, ello no se diferencia fundamentalmente de los criterios que llevaron al pueblo judío ayer, o al pueblo palestino de hoy, o a los distintos grupos étnicos de la antigua Yugoslavia, a reclamar su autodeterminación en virtud de derechos universales.

\section{Educación intercultural en el aula: las Universidades Interculturales en México}

Entre diciembre de 2006 y mayo de 2008 visité seis Universidades Interculturales, dos veces en algunos casos, y participé del Congreso sobre Eduación Superior para Pueblos Indígenas en Patzcuaro en noviembre de 2009. Evidentemente no se trata de una etnografía en profundidad de una institución, pero eso se compensa por los contactos prolongados en una variedad de instituciones y con individuos externos que han jugado un papel importante en su evolución. De esa manera se trató de obtener una visión multifacética de las UI como experimento educacional.

México cuenta con nueve Universidades Interculturales directamente financiados por el estado, más una que pertenece a la red de Universidades Jesuitas. En octubre del 2008 las estatales contaban con 5700 estudiantes de los cuales 2900 estaban en la Universidad Intercultural de Chiapas (UNICH) y la Universidad Autónoma Indígena de Mexico en Sinaloa (UNAIM); después han crecido mucho. Las estatales se encuentran ubicadas, con excepción de la UNICH, en zonas apartadas con significativa población indígena. En los párrafos siguientes voy a centrarme en dos o tres momentos de mi investigación que me permiten indagar lo que realmente se quiere decir por interculturalidad en el aula. 
L'Universidad Intercultural del Estado de Tabasco (UNIET) situé à Oxolotan-Tacotalpa

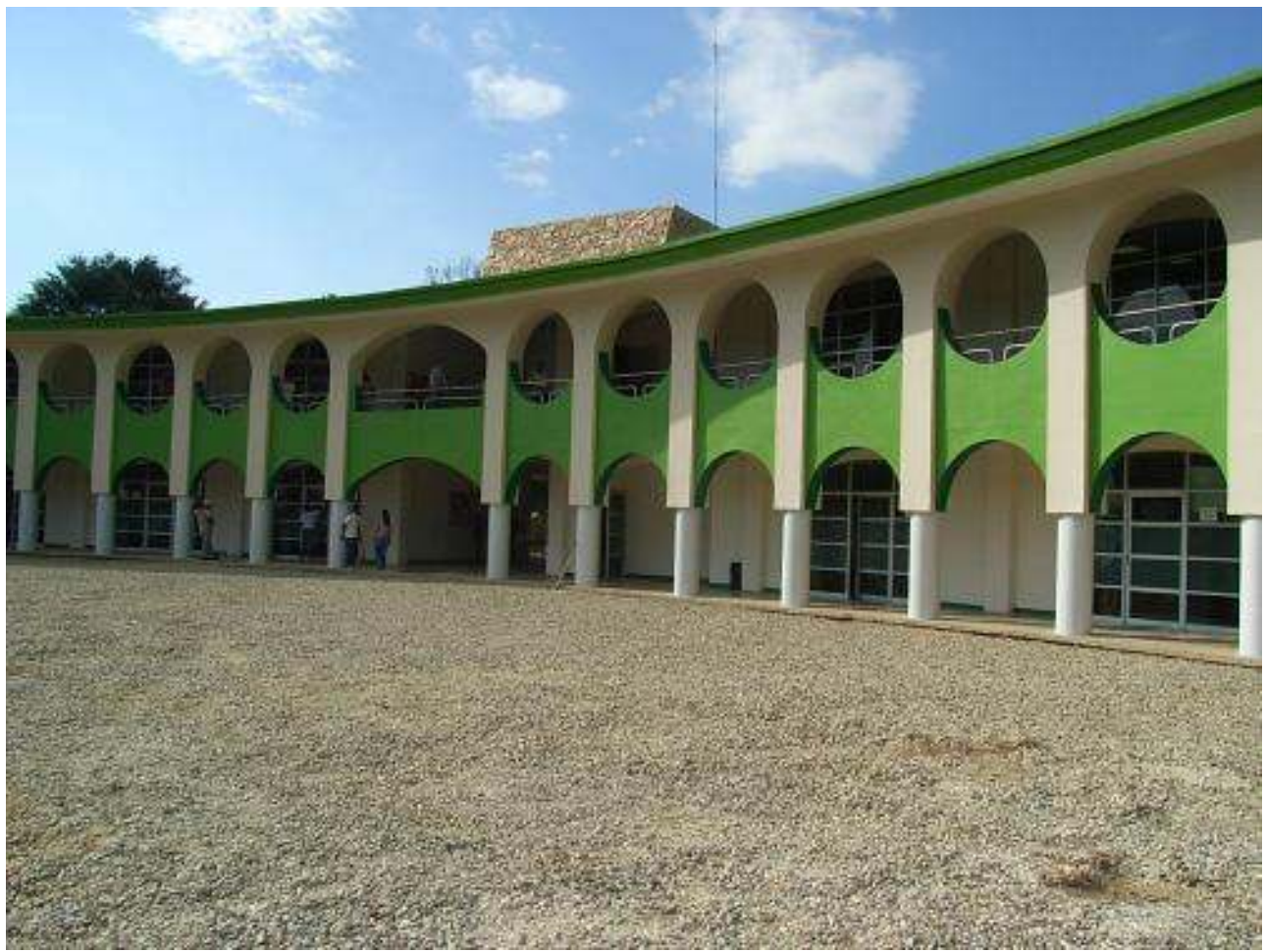

CRÉDITS: D. LEHMANN, 2007

\section{L'Universidad Intercultural del Estado de Tabasco (UNIET) situé à Oxolotan-Tacotalpa}

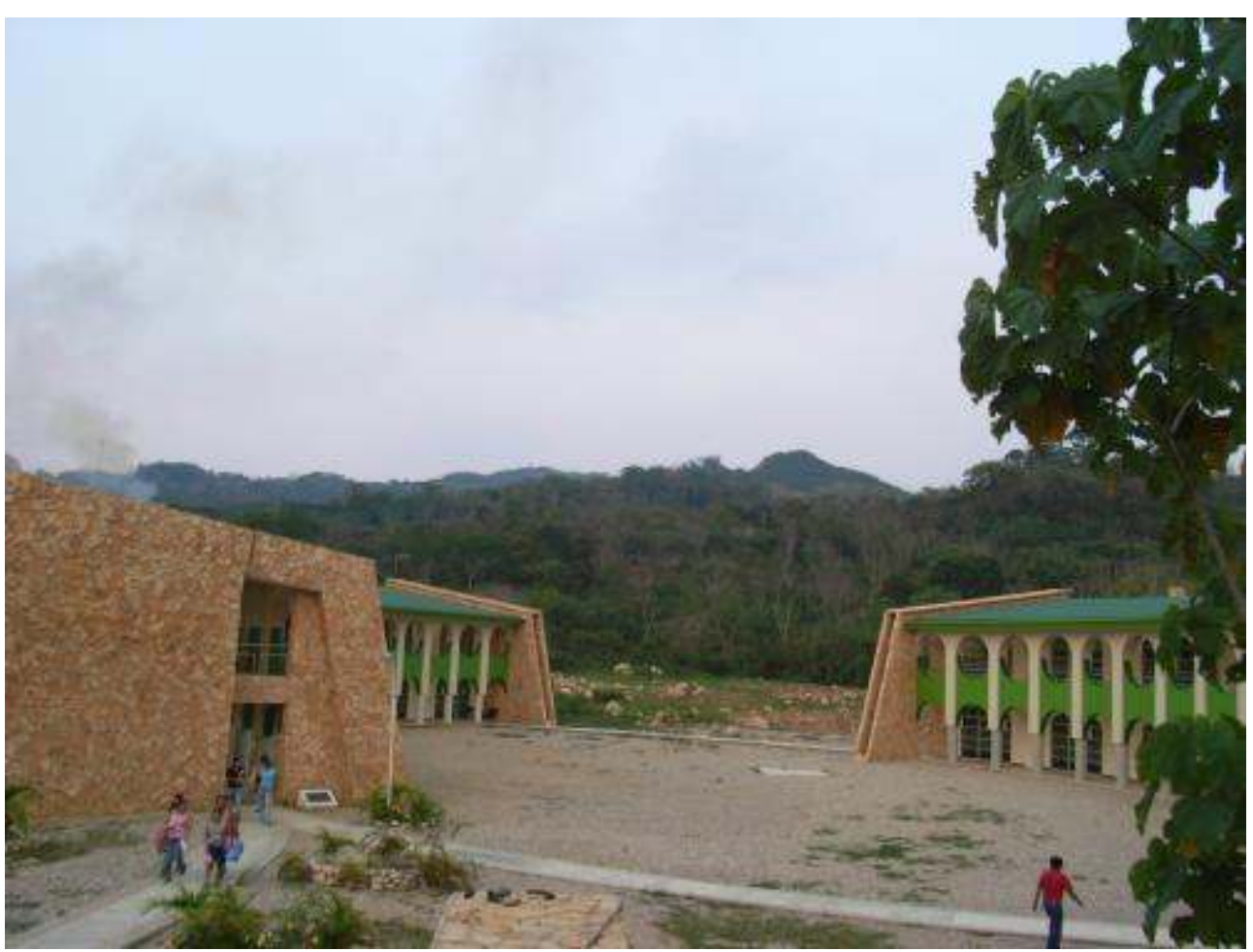

CRÉDITS: D. LEHMANN, 2007 
Une filiale de l'Universidad Veracruzana Intercultural située à Las Selvas, près de Acavucan dans l'état de Veracruz

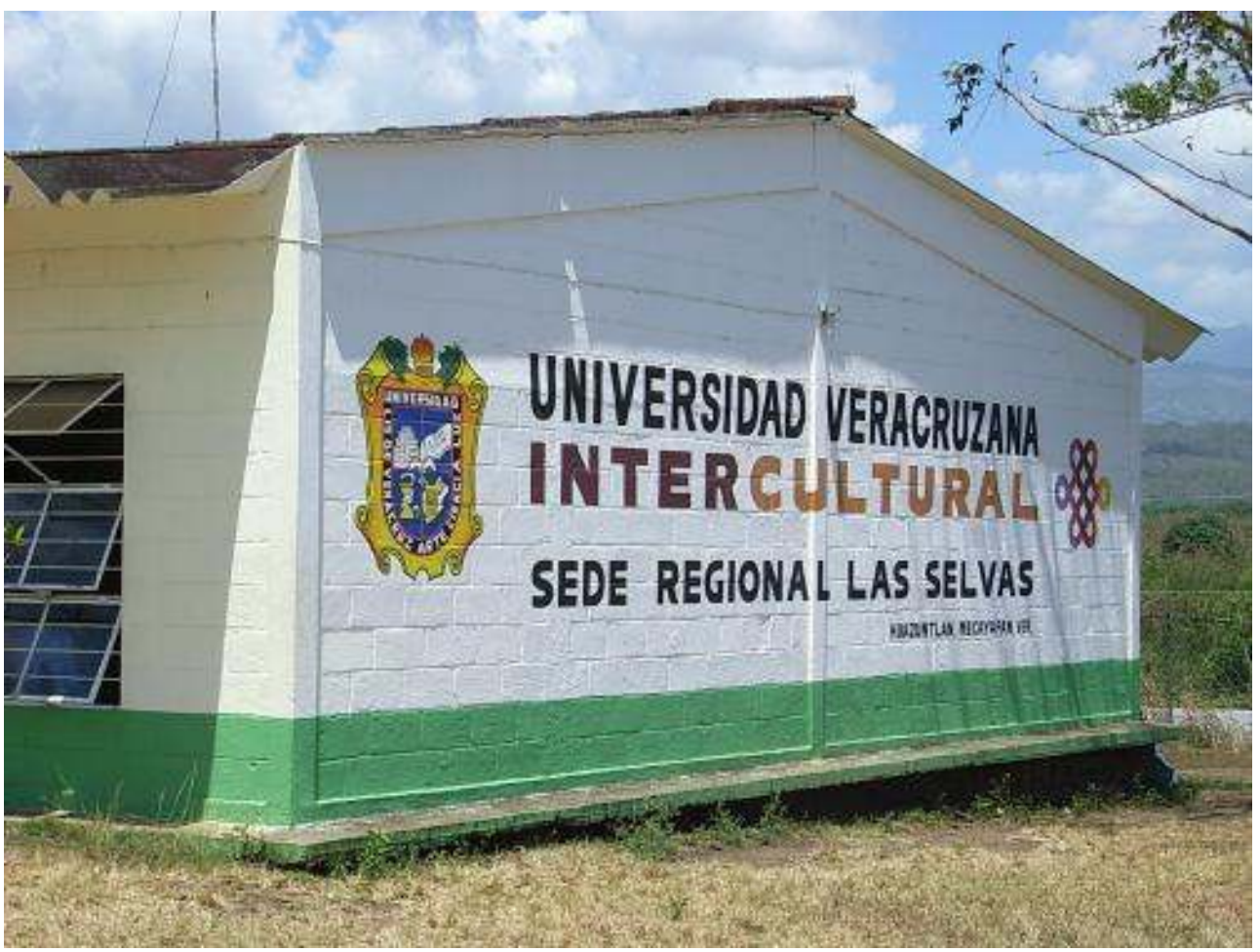

CRÉDITS: D. LEHMANN, 2007

L'Universidad Intercultural de Chiapas (UNICH) à San Cristobal de las Casas

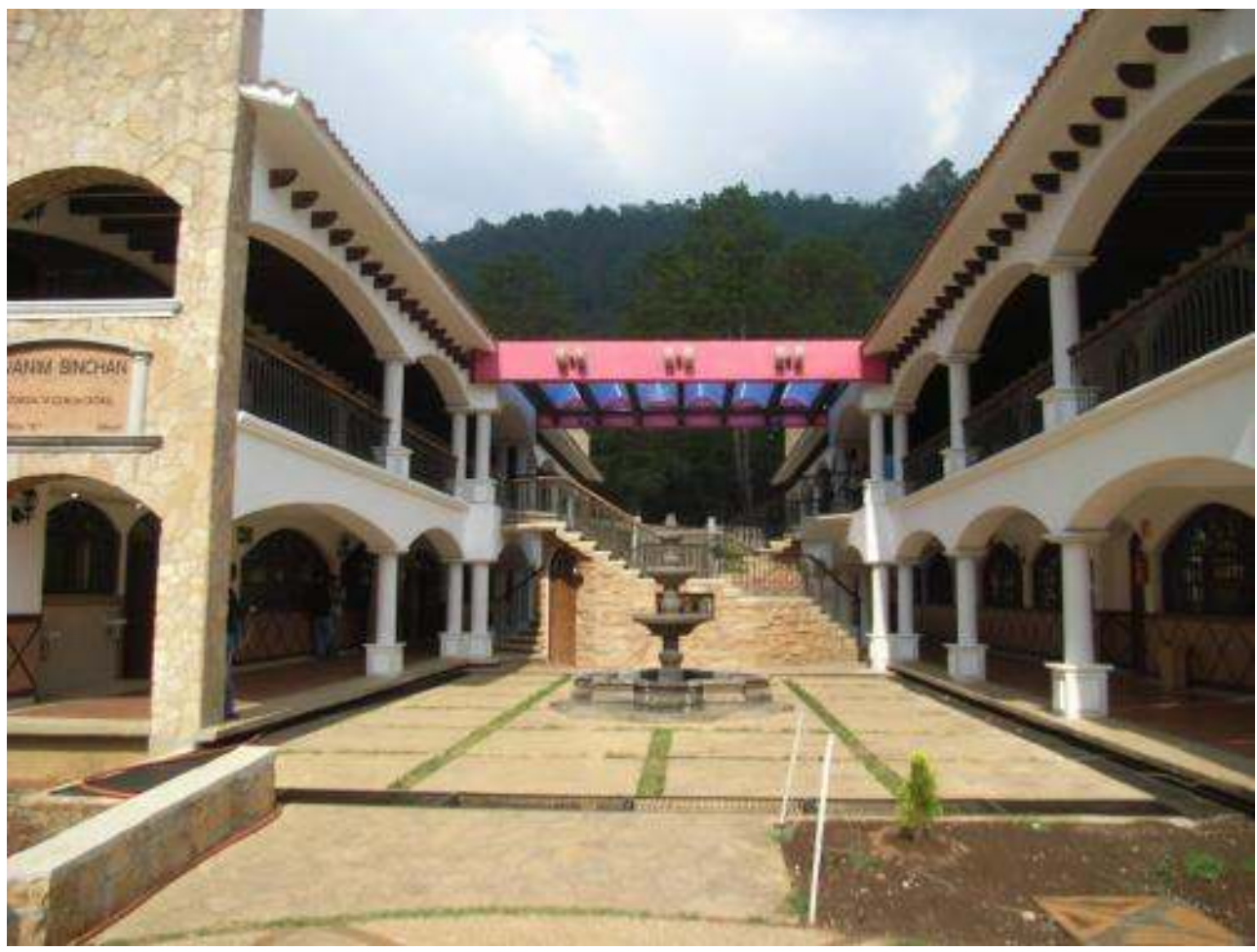

CRÉDITS: D. LEHMANN, 2007 
Durante mi trabajo de campo, me encontré en la situación de actor-interlocutor y además me sentí obligado a una especie de autocrítica. En la Universidad Intercultural del Estado de Tabasco (UNIET), un grupo de profesores me pidieron mi opinión sobre la cuestión 'en qué consiste un ensayo?' - es decir un ensayo escrito como cumplimiento de una tarea académica. En un primer momento contesté como buen profesor europeo (y probablemente un profesor universitario latinoamericano hubiera contestado de la misma forma) que en un ensayo 'no se piden opiniones personales a menos que estén fundadas en información disponible públicamente'. A eso, uno de los profesores contestó recordándome que el conocimiento codificado oficial desvaloriza el conocimiento propio de los estudiantes. Al principio desconfié de la respuesta, pero más tarde he llegado a pensar que mientras mis estudiantes ingleses están acostumbrados desde su niñez a que se les escuche sus opiniones, en el sistema mexicano es consabido que en las escuelas rurales - talvez en las escuelas en general hay una cultura de la clase magistral, y que el maestro, o más frecuentemente la maestra, 'lo sabe todo'. Dadas las 'asimetrías escolares' de las cuales hablaban los profesores, refiriéndose a la educación anterior de sus 'chamacos' (palabra popular para referirse a jóvenes'), se entiende la necesidad de que los que llegan a la universidad habiendo padecido esas asimetrías necesiten antes que nada aprender a expresarse, y a tener una 'voz'. Si a esto se añaden los factores raciales de menosprecio que afectan a estudiantes indígenas, se comprende que la interculturalidad no necesita de elementos relativistas o epistemológicos para empoderar a los alumnos frente a la dimensión colonial de su exclusión, subrayada por Walsh y otros. Me aventuraría más aún: una tentativa incierta de enseñar a niños indígenas su propia lengua como lengua escrita puede infligirles un sentimiento de vergüenza o de humillación si los profesores son mal adiestrados y sobre todo si ellos mismos no se identifican como indígenas o si, siendo de familia o de comunidad indígena, evidencian un esfuerzo de distanciamiento de su patrimonio indígena o de su comunidad o familia de orígen. A nivel de la educación superior eso puede cambiar, tal como pude averiguar de la boca de estudiantes dela UNICH y de la Universidad Intercultural del Estado de México (UIEM) cuyo entusiasmo o interés por sus raíces les había llevado a vencer resistencias y hasta vergüenza imbuidas por sus padres, siendo de familia o de comunidad indígena ${ }^{7}$. En estas condiciones, uno se pregunta si el enfoque constructivista no contribuiría más a las metas de la educación intercultural - de darles a los alumnos la oportunidad de expresarse con su propia 'voz' - que tentativas poco convincentes de enseñanza bilingüe. La duda viene reforzada a la luz de las palabras de Saldívar, porque los que vienen a apoyar la enseñanza formal (con ortografia, gramática etc.) son ONGs internacionales y los profesores suelen ser representantes de la cultura dominante o por lo menos la cultura mestiza más que la indígena - en la medida en que se puede hablar en esos términos de dos conjuntos culturales en perpétua redefinición uno con el otro. Dicho en forma un poco hiriente y exagerada, la educación bilingüe arriesga presentarse como otra imposición de tinte colonial.

\section{Educación Liberadora en las Universidades Interculturales}

En las UI aparte de la dimensión intercultural existe una visible influencia de la ya nutrida tradición de educación popular, fundada en América Latina por Paulo Freire, 
representada mundialmente por el propio Freire y por el pensamiento del psicólogo ruso Vygotsky (1896-1934) y el psicólogo norteamericano Jerome Bruner (n. 1915). En la UAIM, la primera UI reconocida oficialmente e inesperadamente en Sinaloa en 1999, antes de que el gobierno Fox deflagrara la pequeña ola que ahora conocemos, se da una tendencia nueva y el antropólogo Jesús Angel Ochoa-Zazueta y el economista Ernesto Guerra erigen un modelo académico original, lejos de los centros intelectuales del país, y en una universidad bulliciosa en instalaciones bastante precarias en el modestísimo pueblo de Mochicahui. En una entrevista Guerra resumió su filosofía: (a) insatisfacción con los procedimiento jerárquicos y precocinados con los cuales los profesores imparten sus materias; (b) oposición a las formas tradicionales de evaluación que crean competencia innecesaria entre estudiantes e imponen uniformidad cuando 'el examen tiende a uniformizar y nosotros estamos trabajando con la diversidad', (c) los exámenes 'producen el fracaso'; (d) una conciencia aguda del sesgo cultural inherente al sistema de exámenes: 'una cultura no puede reprobar otra cultura...' y (e) un multiculturalismo moderado, porque por un lado dice que 'aquí pensamos que el conocimiento es relativo a cada cultura', pero por otro, inmediatamente después, dice que '...no estamos hablando de saberes indígenas sino de conocimientos indígenas' lo que yo interpreto como una manera de distanciarse del relativismo cultural. Sin embargo, explica que si por ejemplo se está analizando las 'propiedades del árbol...depende de quien este analizando al árbol,... de la cosmovisión de la gente que está analizando... muchas veces pensamos que hay una ciencia universal pero del punto de vista de los Tzotzil, de los Chol, de los Yoremo, el árbol tiene un significando diferente del árbol de nosotros'. Pero no se trata de privilegiar una $u$ otra cultura, sea occidental o no, sino de crear un diálogo entre estudiantes: 'lo que ellos demuestran en la acreditación es una mezcla entre su bagaje cultural y el bagaje cultural de los facilitadores sin decir cuál es el verdadero, sino simplemente es un dialogo intercultural'.

El modelo instalado por Guerra y su mentor Ochoas prescindió del aula y del examen tradicional: los titulares académicos (porque no hay 'estudiantes') buscaban a facilitadores (porque no hay 'profesores'), procedían por investigación, formaban pequeños equipos, y cuando se sentían listos se presentaban a un jurado (un 'sínodo' en la jerga de la UAIM); si el resultado no era satisfaciente se les aconsejaba de volver a presentarse en otra ocasión. O sea, como dice Guerra, 'aquí no hay fracasados'.

Un miembro de un equipo de inspección enviado en el cuadro del PIFI, declaró en entrevista que 'nos quedamos sorprendidos: los estudiantes estaban estudiando bajo los árboles... había colas para ir a presentarse al examen..' [se olvidó de decir 'sínodo'] ... Y describió una escena que parecía inspirada en Jean-Jacques Rousseau: 'no hay materias: el facilitador está con su proyecto consiguiendo alumnos y es el experto en esa materia; en vez de dar clases a intervalos regulares, él está allí y cuando siente que hay un grupo grande hace reuniones con ellos para decirles lo básico, qué tienen que leer...'

41 Aún sin tomar en cuenta ciertas desavenencias político-burocráticas propias del contexto local, el modelo no ha dejado de tener sus costos y también su enemigo en el PIFI y en lo que el PIFI representa como burocratización de la vida académica. Por otra parte, es evidente que si los titulares académicos tienen acceso permanente a sus facilitadores, éstos sufren una presión más fuerte de lo que sería EN un sistema donde la jerarquía controla el horario. Y para las autoridades federales (dueños de la certificación de la institución como universidad) una institución sin exámenes ni profesores ni aulas es como un desafío al sistema reinante. 
42 Así no es sorprendente que para el año 2008 Ochoas había renunciado y Guerra había sido deslocado de su puesto de Coordinador Académico para Director del Pos-Grado. Sin embargo no está claro que el modelo haya sido abandonado: el nuevo Rector, personaje de largo desempeño en cargos burocráticos ligados al PRI sinaloense, me hablaba con toda la jerga del modelo y se batió con éxito para salvar la universidad. Además, muchos aspectos del modelo aparecen en otras universidades (la UIET de Tabasco por ejemplo) donde pude notar un ambiente de fraternidad entre alumnos y profesores y donde además la terminología de facilitadores, o asesores, por ejemplo, había filtrado. En un ambiente más parecido a una universidad tradicional como la UNICH no se notaba eso.

Las UI han traído una forma inusitada de interacción a la educación superior estatal mexicana, donde ha prevalecido la clase magistral, el profesor omnisciente y un estudiantado de masa: en ellas la interacción es mucho menos distante, menos apresurada, más íntima si se quiere (con la excepción de la UNICH). Esta innovación se debe a su ubicación en zonas apartadas, al hecho que la brecha etaria o la distancia social entre los miembros del cuerpo docente ('facilitadores', o 'asesores' en más de un caso) y los estudiantes son menores que en las universidades tradicionales, y que aquéllos tienden a tener cierto compromiso político con su trabajo y sus estudiantes.

Otro rasgo que refleja la influencia de la educación popular es la importancia de la investigación en los cursos de las UI. Fiel, aparentemente, al legado de Paulo Freire, en todas las universidades visitadas los alumnos arrancan haciendo investigación casi desde el inicio de sus estudios. La investigación de su propia realidad fue una piedra angular de los proyectos de alfabetización con adultos que el maestro desarrollaba en Brasil y Chile durante los años 60 , porque decía que la alfabetización consistía en adquirir una conciencia crítica, no simplemente asimilar como leer o escribir según las instrucciones de un enseñante - de allí la palabra concientización. Para los alumnos de las UI, inevitablemente, muchas veces la investigación es un proyecto en sus propios pueblos.

Todo esto se resume en la palabra clave 'vinculación', que tiene mucha resonancia en la educación superior intercultural. Se refiere a las actividades vinculadas con las comunidades de origen de los estudiantes, y al deseo de crear lazos por los cuales las UI colaboren al desarrollo de las comunidades. De alguna manera, la vinculación, es decir la investigación por los alumnos de una realidad que les pertenece a ellos más que a sus profesores, comporta una relación parecida a la pretensión de los entusiastas de la educación bilingüe: coloca a los educandos en una relación 'enajenada' (en el sentido brechtiano) de observador o estudioso de algo que le pertenece a ellos mismos, y una realidad que les es propia se vuelve un teatro donde son ellos los espectadores y los críticos. En el caso de la lengua los expertos (Zavala 2002; Zavala 2007; Lopez 2009), no nos traen noticias muy alentadores; en el caso de la vinculación no hay diagnósticos, pero por lo menos el objetivo es más realista.

\section{Conclusión}

En un juicio famoso, la Corte Constitucional de Colombia justificó, en 1996, el uso del látigo en el castigo impuesto por un juez indígena, con varios argumentos, entre ellos que se trata de 'una figura simbólica o, en otras palabras, un ritual que utiliza la comunidad para sancionar al individuo y devolver la armonía... y que el sufrimiento 
que esta pena podría causar al actor, no reviste los niveles de gravedad requeridos para que pueda considerarse como tortura, pues el daño corporal que produce es mínimo.' (Corte Constitucional de Colombia, Sentencia No. T-523/97: Principio de Diversidad Etnica y Cultural) $)^{8}$. Por otra parte, frente al argumento de que el fuete no era originario ni auténtico porque había sido introducido durante el período colonial, la Corte anotó que la norma vigente 'no exige que las prácticas y procedimientos deban ser llevadas a cabo de la misma manera que como lo hacían los antepasados, porque el derecho de las comunidades indígenas, como cualquier sistema jurídico, puede ser dinámico'.

Traigo a luz este pronunciamiento inusitado porque ello no se diferencia fundamentalmente de la interculturalidad que hemos descrito para los casos de la educación blingüe y para la vinculación. Es decir, introduce elementos de erudición moderna, o de contextualización socio-histórica que interponen una distancia reflexiva frente a prácticas que suelen ser posicionadas como parte de lo impensado o de lo irreflexivo de la tradición auténtica. Pero en cada uno de esos casos - el aprendizaje de la lengua 'propia', la vinculación, y la decisión del juez Gaviria - se introduce el espacio de reflexividad y de enajenación, con lo cual el orden indigena es incorporado al orden moderno. El problema es que, mientras para algunos, ese orden moderno es neoliberal y colonial, es difícil imaginar un proyecto liberador sin ello.

\section{BIBLIOGRAPHIE}

Baronnet B., "La question de l'interculturalité dans les expériences d'éducation en terres zapatistes", in C. Gros and D. D. Kervran (Dir.), Le multiculturalisme au concret: un modèle latinoaméricain?, Paris, Presses Sorbonne Nouvelle, 2011.

Bartra R., La sangre y la tinta: ensayos sobre la condición postmexicana. Mexico DF, Océano, 1999.

De la Peña G., "Social and Cultural Policies toward Indigenous Peoples: Perspectives from Latin America", Annual Review of Anthropology, vol. 34: n¹, 2005, p. 717-739.

Dutcher N., Expanding educational opportunity in linguistically diverse societies, Washington DC, Centre for Applied Linguistics, 2002.

Lopez L. E., De resquicios a boquerones: la Educación intercultural bilingüe en Bolivia, La Paz, PROEIBAndes/Plural, 2005.

Lopez L. E., "Reaching the unreached: indigenous intercultural bilingual education in Latin America", Background paper prepared for the Education for All Global Monitoring Report 2010, UNESCO, 2009.

Lopez L. E., "Reaching the unreached: indigenous intercultural bilingual education in Latin America", Background paper prepared for the Education for All Global Monitoring Report 2010/ED/EFA/ MRT/PI/29, UNESCO, 2009.

Navarro Martinez S. and A. Saldívar Moreno, "Construcción y significado de la interculturalidad en la escuela normal indígena intercultural bilingüe "Jacinto Canek»", Revista Pueblos y Fronteras digital, vol. 6: $\mathrm{n}^{\circ} 12,2011-2012$. 
Noel B., "Language, power and schooling: Bolivia's education reform program", College of Education and Human Development, George Mason University, 2006.

Rappaport J., Intercultural utopias: public intellectuals, cultural experimentation, and ethnic pluralism in Colombia, Durham, Duke University Press, 2005. (Spanish trans,: Utopías Interculturales: Intelectuales Públicos Experimentos Con La Cultura y Plural, Universidad del Rosario, 2008)

Rappaport J., Utopías Interculturales: intelectuales públicos, experimentos con la cultura y pluralismo étnico en Colombia, Bogotá, Universidad del Rosario, 2008.

Saldívar A., "Diseno de estrategias socioeducativas para el desarrollo: acercando la educacion al desarrollo en Chiapas", Cultura y Educación, vol.13, 2001, p. 21-36.

Saldívar Moreno A., M. M. Micalco Mendez, et al., "Los retos en la formación de maestros en la educación intercultural", Revista Mexicana de Investigación Educativa, vol. 9: n²0, 2004, p.109-128.

Sierra M. T., "Las mujeres indígenas ante la justicia comunitaria: perspectivas desde la interculturalidad y los derechos", Desacatos (CIESAS), n³1, 2009, p. 73-88.

Walsh C., "Políticas y significados conflictivos", Nueva Sociedad, vol. 165, 2000, p.121-133.

Walsh C., "(De) Construir la interculturalidad: Consideraciones críticas desde la política, la colonialidad y los movimientos indígenas y negros en el Ecuador", in N. Fuller (ed.), Interculturalidad y Política. Desafíos y posibilidades, Lima, Red para el Desarrollo de las Ciencias Sociales en el Perú, 2002, p.115-142.

Zavala V., Desencuentros con la escritura: escuela y comunidad en los Andes peruanos, Lima, Red para el Desarrollo de las Ciencias Sociales en el Perú, 2002.

Zavala, V., "Una mirada a la formación docente en educación bilingüe intercultural en la zona andina del Perú", in R. Cuenca, N. Nucinkis and V. Zavala (eds.), Nuevos maestros para América Latina, Madrid, Ediciones Morata, 2007.

Žižek S., "Multiculturalism, Or, the Cultural Logic of Multinational Capitalism", New Left Review, vol. 1: n²25, 1997, p.28-51.

\section{NOTES}

1. El libro trata del CRIC - Consejo Regional Indígena del Valle del Cauca - un conjunto de organizaciones fundado en 1971, que fue talvez el primer impulsor de la ola de indígenismos del último cuarto del siglo XX. En el contexto de su libro (que sólo he leído en su versión inglesa) no se puede hablar de 'entrevistados' ni de 'informantes' porque la autora es a la vez asesora, profesora, animadora, y de alguna manera portavoz del movimiento o de algunas de sus corrientes. Rappaport logra equilibrar cierta desconfianza frente al esencialismo con un compromiso en la lucha contra la exclusión..

2. ver el ensayo titulado Violencias salvajes: usos, costumbres y sociedad civil. Bartra monedó la famosa frase 'kitsch tropical' en aras del levantamiento zapatista en 1994.

3. www2.sepdf.gob.mx/info dgose/texto.

4. Este documento así como el citado anteriormente parecen haber sido retirados del sitio de la CGEIB.

5. Cuando Freire trabajó en Chile con la Reforma Agraria en los años 60 (después de huir del Brasil en pos del golpe de 1964, su método fue adoptado con resultados aparentemente muy positivos para la movilización campesina, y la palabra 'realidad' se repetía siempre en el contexto de la educación de adultos propicidos por el gobierno. Se dijo que el entonces Presidente Frei 
acabó muy preocupado por los efectos del famoso 'método de Freire' y presionó para que la ONU no renovara su contrato. Freire entonces se fue a Harvard.

6. ver el site http://catherine-walsh.blogspot.co.uk/

7. En el caso de la formación de profesores de EIB en la Sierra peruana, a pesar de un creciente interés por parte de alumnos en aprender 'su' lengua, había una gran escasez de formadores con un 'dominio del quechua que les permita conducir una sesión de aprendizaje en esta lengua' . El mismo libro incluye un capítulo de Elba Gigante sobre 'Diversidad sociocultural y formación docente en México', pero infelizmente trata sólo de la formación para la educación indígena, que no es lo mismo que la educación intercultural. Sólo da informaciones formales y oficiales que no ofrecen un análisis de la realidad de la educación bilingue.

8. http://www.corteconstitucional.gov.co/relatoria/1997/T-523-97.htm

\section{RÉSUMÉS}

Cet article étudie les différentes significations associées au concept d' interculturalidad et leur mise en application dans les Universidades Interculturales depuis leur fondation par le gouvernement mexicain en 2000. S'appuyant à la fois sur des entretiens menés dans plusieurs Universidades Interculturales entre 2006 et 2010 et sur un rappel du contexte historique, cette analyse démontre que le fonctionnement pratique de ces universités trouve tout autant son inspiration dans le courant d'éducation populaire initié notamment par Paulo Freire dans les années 1960 que dans l'interculturalidad. Elle soutient aussi qu'il faut voir dans ce nouveau type d'institutions aussi bien une forme de discrimination positive qu'une expérience dans le domaine du multiculturalisme.

This paper explores the many meanings associated with the concept of interculturalidad and their application in the case of the Universidades Interculturales established by the Mexican government since 2000. On the basis of interviews carried out in several Universidades Interculturales in the period 2006-2010, and of much background information, the analysis shows that the practice of the universities is inspired as much by the popular education tradition of Paulo Freire and others as by interculturalidad, and that they can be seen as a type of affirmative action as much as a venture in multiculturalism.

Este artículo explora las distintas significaciones y puestas en práctica del concepto Interculturalidad por las Universidades Interculturales establecidas por el gobierno Mexicano desde 2000. Basándose en múltiples fuentes de información como en entrevistas realizadas en varias Universidades Interculturales entre 2006 y 2010, el análisis concluye que las prácticas de las Universidades se inspiran tanto en la tradición de la educación popular sudamericana elaborada por Paulo Freire y otros en los años 1960 como en la Interculturalidad. El dispositivo puede ser interpretado como una forma de discriminación positiva y como un intento de multiculturalismo. 


\section{AUTEUR}

\section{DAVID LEHMANN}

David Lehmann ha enseñado en la Universidad de Cambridge desde 1973. Es autor de Struggle for the Spirit: religious transformation and popular culture in Brazil and Latin America (1966) y (con Batia Siebzehner) de Remaking Israeli Judaism: the challenge of Shas (2006), y de numerosos artículos sobre la religion y la globalización y sobre el campo religioso en América Latina. El presente artículo es fruto de un proyecto financiado por la British Academy titulado Multiculturalism in Latin America: a study in the diffusion of ideas. 